\title{
A Silent Emergence of Culture: The Social Tuning Effect
}

\author{
Garriy Shteynberg \\ Northwestern University
}

\begin{abstract}
Scholars have long been concerned with understanding the psychological mechanisms by which cultural (i.e., shared) knowledge emerges. This article proposes a novel psychological mechanism that allows for the formation of cultural memories, even when intragroup communication is absent. Specifically, the research examines whether a stimulus is more psychologically and behaviorally prominent when it is assumed to be experienced by more similar versus less similar others. Findings across 3 studies suggest that stimuli such as time pressure (Study 1), words (Study 2), and paintings (Study 3) are more psychologically and behaviorally prominent when they are thought to be experienced by more (vs. less) similar others. Critically, the effect is absent when similar others are thought to be experiencing distinct stimuli from the participant (Study 3). Taken as a whole, these results are consistent with the hypothesis that stimuli which are assumed to be experienced by one's social group are more prominent in both cognition and behavior. Theoretical implications for the emergence of culture are discussed.
\end{abstract}

Keywords: culture, social tuning, social influence, social memory, social identity, social knowledge

Culture is a ubiquitous concept in the social sciences that purports to capture something essential about human life. It is also one of the more nebulous constructs in scholarly discourse, encompassing at least 164 separate definitions (Kroeber \& Kluckhohn, 1952). Perhaps, in its most provocative incarnation, culture stands for the human ability to form social groups in which shared understandings of what is true, good, and real are constituted. Indeed, the notion that humans belong to distinct social groups that cultivate and maintain unique patterns of knowledge is both the lynchpin of social science scholarship (Durkheim, 1893/1984; James, 1907; Lewin, 1943; Mead, 1934; Vygotsky, 1978) as well as a honed intuition of a veteran traveler. The outstanding question for culture scholars, however, is how to account for group members' capacity to achieve and maintain coordinated knowledge of their environment. Put differently, what is the set of social and psychological factors that allows for the emergence of cultural or shared knowledge?

One generally accepted theory in social psychology is that humans are exceptionally adept at aligning personal attitudes with

This article was published Online First August 16, 2010.

Garriy Shteynberg, Kellogg School of Management, Northwestern University.

This research was facilitated by a National Science Foundation Graduate Research Fellowship and a U.S. Department of Homeland Security START Dissertation Fellowship. I would like to thank Michele J. Gelfand, Arie Kruglanski, Rebecca Ratner, Charles Stangor, Paul Hanges, Harold Sigall, Janetta Lun, Lisa M. Leslie, Bibb Latané, Andrzej Nowak, and Curtis Hardin for comments on a version of this article. I would also like to acknowledge my research assistants Samuel Birk and Ava Safaie for help with data collection and my wife Catherine R. Shteynberg for editorial assistance.

Correspondence concerning this article should be addressed to Garriy Shteynberg, Kellogg School of Management, Northwestern University, 2001 Sheridan Road, Evanston, IL 60208. E-mail: gshteynberg@gmail.com those of their social groups (Festinger, 1957). Indeed, a number of studies confirm that when individuals are aware of another group member's attitude, their own view will gravitate toward that of their group member (Asch, 1952; Echterhoff, Higgins, \& Levine, 2009; Sechrist \& Stangor, 2001; Turner, 1991, 1999). This finding supports the notion that the emergence of shared knowledge is a result of the human propensity to "tune into" the attitudes of one's social group (Hardin \& Higgins, 1996).

Yet attitudes constitute a unique form of knowledge that is inherently evaluative. Importantly, we do not yet know whether nonevaluative forms of knowledge, such as basic memories for stimuli in one's environment, are also a product of social tuning. Indeed, the seminal theoretical view in social psychology maintains that attitudes are uniquely susceptible to social influence because they are experienced as subjective and uncertain (Festinger, 1950), which in turn implies that "tuning into" the perspective of one's group is primarily driven by the need for certainty in one's judgments.

Alternatively, I propose that humans are driven not only to attain certain knowledge but also to prioritize acquiring knowledge that is coordinated with their social group. It follows that individuals' nonevaluative and more certain forms of knowledge, such as basic memories for stimuli, should also be shaped by their group's perspective. More specifically, I posit that people "tune into" what they assume to be the stimuli experienced by their group, thereby achieving greater social coordination. Notably, unlike burgeoning research that implicates intragroup communication in the emergence of shared knowledge (see Hirst \& Echterhoff, 2008, for a review), I examine how assumptions about group members' experiences shape cognition and behavior. More specifically, three studies explore whether stimuli such as time pressure, words, and paintings are rendered more prominent in cognition and behavior when participants assume that their group is experiencing these stimuli. In what follows, I elaborate on my theoretical stance and introduce data that provide support for the hypothesis. 


\section{Why Would Individuals Socially Tune Their Memories?}

A deluge of environmental stimuli is perpetually available for human cognitive consumption. Faced with limited processing and memory resources (Broadbent, 1958; Miller, 1956), the human brain must mentally prioritize certain stimuli over others. Although this choice may be guided by any number of active goals, there are good reasons to believe that humans should mentally prioritize those stimuli in their environment that are assumed to be experienced by their group.

First, evolutionary psychologists have argued that like biological structures, a select number of human motives are also a result of evolution by natural selection and can be traced to the selection pressures faced by early hominids in the Pleistocene epoch (Cosmides, Tooby, \& Barkow, 1992). If we agree that individual survival relied on successful execution of social activities such as food gathering, game hunting, shelter maintenance, and enemy repulsion (Wilson \& Wilson, 2007), it follows that group members were under constant pressure to develop shared mental representations of their environment. Put differently, those group members that were more likely to attain shared memories had a greater chance of survival and reproduction. For instance, greater mental prioritization of a location experienced by fellow group members would have led to more efficient coordination of social action within that location. In sum, it is likely that psychological adaptations that enhanced memory attunement to one's social group would enhance both individual-level and group-level fitness (Sober \& Wilson, 1998).

Second, as reviewed, social psychological scholarship has convincingly shown that individuals align their attitudes with those of their social group (Echterhoff et al., 2009). It is a truism to say that attitudes must have a referent-a stimulus around which evaluative consensus occurs. However, it is not commonly recognized that reaching evaluative consensus about a stimulus is much more likely if group members overlap in their stimuli memory to begin with. For instance, in the course of a conversation, group members are likely to reach evaluative consensus on a greater number of stimuli if at the beginning of that conversation they overlap on what is on top of their minds. In sum, creating shared memories facilitates the subsequent creation of shared attitudes that concern those memories.

\section{How Do Individuals Socially Tune Their Memories?}

To the extent that the creation of shared memories is a priority, individuals will look for environmental cues that with some probability predict the stimuli that their group members are experiencing. Indeed, the developmental literature suggests that at as soon as 9 months of age, infants follow eye gaze and engage in prolonged instances of triadic attention, involving multiple people directing their attention toward a common object (Baron-Cohen, 1995; Tomasello, Carpenter, Call, Behne, \& Moll, 2005). Thus, already at this early stage of development, humans purposefully orient themselves to the stimuli experienced by others. Moreover, there is some evidence that such social attunement is an important antecedent of language development (Carpenter, Nagell, \& Tomasello, 1998; Morales et al., 2000). Of course, eye gaze is not the only environmental cue that predicts what our group is experiencing.
Other cues include pointing movements (Clark, 2003), verbal communication (Clark, 1996), and, as I discuss next, abstract thought that is informed by environmental cues and prior knowledge.

As humans develop, they join a greater number of social groups whose membership is more and more ecologically diffused-from families and friends to political parties and nation-states. As such, environmental cues such as eye gaze and pointing can no longer suffice as the only predictors of what the social group experiences. However, with the development of abstract thought, individuals can combine environmental cues and prior knowledge to construct reasonable assumptions about which stimuli their social groups are experiencing. For instance, when consuming stimuli through a television, a newspaper, or an Internet site, an individual is able to implicitly gauge whether his or her group members are also experiencing or have experienced the same information.

I hypothesize the following: Stimuli that are assumed to be experienced by one's social group have greater cognitive accessibility. The cognitive accessibility of a stimulus refers to the readiness with which a mental representation of that stimulus is employed in cognitive operations (Bruner, 1957; Higgins \& King, 1981). Notably, this hypothesis implies that even in the absence of intragroup communication, group members will develop shared memories. That is, if for Group Member A, Stimulus X is more cognitively accessible because he or she correctly assumes that Group Member B has experienced it, and likewise, if for Group Member B, Stimulus X is more cognitively accessible because he or she correctly assumes that Group Member A has experienced it, then Group Members A and B establish a shared memory for Stimulus X.

\section{Overview of Studies}

The above hypothesis was examined across three studies. Because it was necessary to manipulate the extent to which participants assumed that their group members were also experiencing a given stimulus, I faced the choice of how to manipulate the level of group identification with other observers. On the basis of the voluminous social categorization literature, I manipulated group identification by varying the level of others' similarity to the self (Turner, 1999; Turner, Hogg, Oakes, Reicher, \& Wetherell, 1987). As such, it was expected that when the stimuli were assumed to be experienced by more (vs. less) similar others, the stimuli would be more (vs. less) cognitively accessible.

This key hypothesis was tested across a variety of stimuli, the cognitive accessibility of which was measured in several ways. For instance, I examined whether time pressure that was assumed to be experienced by more (vs. less) similar others was more cognitively accessible by measuring the speed with which participants arrived at a decision (Study 1). In a more direct test of the hypothesis, I examined whether words that were assumed to be experienced by more (vs. less) similar others were more cognitively accessible by measuring subsequent word recognition performance (Study 2). Finally, I examined whether paintings that were assumed to be experienced by more (vs. less) similar others were more cognitively accessible by measuring subsequent painting recognition performance (Study 3).

In line with the theory outlined, in Study 3 I operationalized a critical distinction between the assumption that more (vs. less) 
similar others are actually experiencing the same stimuli, and the assumption that more (vs. less) similar others are present but are not experiencing the same stimuli. In the latter condition, the study instructions implied that more similar others performed the same general task but saw different stimuli from those that were seen by the participants. It was expected that only those stimuli that participants thought were experienced by more similar others would be more cognitively accessible.

\section{Study 1}

\section{Method}

Participants were 20 students in a psychology class that were randomly assigned to one of two groups, with each group occupying a separate room. In one of the rooms, participants were told that according to their performance on a scale completed in the previous week, each of them was similar to the others in thinking style. Participants in the other room were not told anything about the others in the room. After the similarity manipulation, all participants were told that they would be shown a slide that described the actions of a paramilitary group and that each of them would be asked to judge whether the paramilitary group was a terrorist organization.

All participants were given a deadline of 5 min to make their judgment. This was further represented by a timer, which was located at the top of the slide that described the paramilitary group. The participants were asked to turn over the response sheet in front of them when they arrived at a judgment of the paramilitary group. The response sheet asked the participants to write down the number of seconds displayed on the timer and then indicate whether they believed the paramilitary group was a terrorist organization.

Of primary interest was how fast the participants across conditions made their decision. It was expected that the time pressure experienced by participants in the more (vs. less) similar others condition would be more cognitively accessible, which would lead to faster judgments. Importantly, participants were not allowed to communicate with one another.

\section{Results and Discussion}

Results indicated that participants in the more similar others condition made their judgments, on average, 37 s quicker $(M=$ $136 \mathrm{~s}, S D=17.13$ vs. $M=173 \mathrm{~s}, S D=46.92$ ). After a $\log$ transformation of the time measure, an analysis of variance (ANOVA) indicated that the difference was statistically significant, $F(1,18)=5.37, p<.05, \eta^{2}=.23$. A $t$ test (equal variance not assumed) confirmed the significance of the results, $t(12.71)=$ $-2.32, p<.05$. In sum, the results suggest that participants in the more (vs. less) similar other condition made quicker decisions. This constitutes preliminary evidence for the hypothesis that stimuli which are assumed to be experienced by more (vs. less) similar others are more cognitively accessible.

\section{Limitations}

There are several alternative explanations for this initial finding. First, it is also possible that priming thoughts of one's thinking style primes self-efficacy in judgment making and perhaps in- creases judgment speed. Second, because participants completed the timed task in one another's presence, it is possible that their responses were influenced by observing one another's actions (i.e., turning over the response sheet), thereby violating the assumption of statistical independence, resulting in the underestimation of standard errors. Third, it can be argued that participants in the more (vs. less) similar others condition perceived greater opinion consensus between themselves and others, resulting in less worry about potential social comparisons and yielding a more confident and/or more comfortable psychological state, which in turn would make for faster cognitive processing. Finally, it is possible that the mere presence of more (vs. less) similar others elevated mood, arousal, or competitiveness that would result in faster decisions.

In the two studies that follow, these alternative explanations are challenged. First, these studies utilize a minimal manipulation of more (vs. less) similarity based on the choice of avatar color. Specifically, in the more similar condition, participants are joined by others who pick the same avatar colors as them, whereas in the less similar condition, participants are joined by others who pick different avatar colors from them. Utilizing this minimal manipulation of others' similarity, I avoided evoking strong social identities such as gender, ethnicity, or nationality. Because the number of cognitions related to minimal identities, such as avatar color group, is lower, it is less likely that identity-specific cognitions could account for the results (Turner, 1999). Second, in the studies that follow, participants are separated across different rooms, and they are thus unable to observe the behavior of others. Consequently, participants cannot influence one another through nonverbal communication channels, assuring response independence. Finally, through additional measures and manipulations, I examine the possibility that participants in the more (vs. less) similar condition perceived greater subjective consensus with others or experienced greater positive/negative affect, greater arousal, or greater competitiveness.

\section{Study 2}

\section{Method}

Participants and general design. Participants were 43 undergraduates $(72.1 \%$ women, $27.9 \%$ men; mean age $=19.19$ years $)$. The study explored whether words were more cognitively accessible when more (vs. less) similar others experienced them. Target word recognition latency (Fazio, 1989) as well as word recognition accuracy served as dependent variables that measured the cognitive accessibility of words.

Procedure and similarity manipulation. Participants arrived at the laboratory three at a time and were placed into separate rooms, with each individual seated in front of a computer. Participants were informed that they would be asked to perform a number of different tasks, such as forming opinions and playing word games. To begin the study, participants were instructed to click on one of five colored avatars on the computer screen. On the screen that followed, participants were asked to judge a painting, which served as a distractor task. Simultaneous with the instructions for the distractor task, participants in the more similar others condition saw that two other participants chose the same avatar colors as they did, whereas participants in the less similar other condition saw that all participants chose unique avatar colors. In 
reality, the others' avatar colors were controlled by the computer to either match the participant's color choice or mismatch it. Participants were made aware that others were experiencing the stimuli presented through instructions and "waiting for others" screens. The latter appeared several times during the study. Finally, participants were also told that their identities and responses would remain confidential.

Notably, the distractor task (i.e., judgment of the painting) also served as a subjective consensus check. That is, participants were asked not only whether they liked the painting but also whether the other participants liked the painting $(1=$ definitely not, $11=$ definitely yes). Accordingly, the absolute value of the difference between the self and perceived others' opinions indicated assumed subjective consensus on the painting task.

The remainder of the study consisted of a signal detection task in which participants were briefly presented with a list of nine five-letter words, which served as the signal. Participants were then presented with a second series of words, some of which they had seen (signal) and others they had not (noise). Participants were asked to identify whether each word had already appeared or was new.

\section{Results}

To test the effect of condition on speed of correct word recognition (speed of signal detection), I calculated participants' average time to recognize the signal correctly. After a log transformation of correct recognition times, a between-subjects ANOVA indicated a significant effect of condition on the speed of signal detection, $F(1$, $41)=8.11, p<.01, \eta^{2}=.17$. As expected, participants in the more similar others condition had faster recognition times $(n=19$; $M=1,079.25 \mathrm{~ms}, S D=301.95)$ than participants in the less similar others condition ( $n=24 ; M=1,558.36 \mathrm{~ms}, S D=814.12$ ). An independent samples $t$ test (equal variances not assumed) confirmed the finding, $t(39.96)=-2.98, p<.01$. To test the effect of condition on accuracy of word recognition, I calculated $d^{\prime}$ ( $Z$ hit rate $-Z$ false alarm rate; Banks, 1970). A between-subjects ANOVA indicated a significant effect of condition on accuracy of word recognition, $F(1,41)=5.75, p<.01, \eta^{2}=.12$, with participants in the similar others condition exhibiting greater accuracy $(n=19 ; M=0.68, S D=1.20)$ than participants in the less similar others condition $(n=24 ; M=-0.54, S D=1.94)$. An independent samples $t$ test (equal variances not assumed) confirmed the finding, $t(38.97)=2.53, p<.01$.

A between-subjects ANOVA indicated that assumed subjective consensus on the painting task did not significantly differ across conditions, $F(1,41)=0.06, p=.80$. That is, participants in the more similar others condition did not perceive greater agreement with others $(n=19 ; M=2.11, S D=1.85)$ than participants in the less similar others condition $(n=24 ; M=1.96, S D=1.97)$.

\section{Post Hoc Analyses}

A more in-depth analysis of hit rates and false alarm rates showed that the difference in the overall accuracy between conditions was attributable to a higher proportion of hits in the more (vs. less) similar others condition ( hit rate $=72 \%$ vs. $60 \%$ ), $t(40.80)=$ $2.22, p<.05$, as well as a lower proportion of false alarms in the more (vs. less) similar others condition (false alarm rate $=7 \%$ vs. $22 \%), t(34.08)=-2.12, p<.04$.

\section{Discussion}

Both word recognition latency and word recognition accuracy results indicated that words were more cognitively accessible for participants in the more (vs. less) similar other condition. As such, the results were consistent with the social tuning hypothesis, where stimuli that are assumed to be experienced by more (vs. less) similar others are more cognitively accessible. Study 2 findings are particularly important due to the use of more direct cognitive accessibility measures.

Notably, Study 2 results challenge several alternative explanations proposed. First, given the minimal similarity manipulation utilized, it is highly unlikely that identity-specific cognitions drove the results. Second, because participants were separated across rooms, the results cannot be due to participants' observations of one another's behavior. Third, given that there were no differences in assumed subjective consensus across conditions, it is unlikely that there were systematic differences in social comparison concerns, felt confidence, and/or comfort. Finally, a simple drive account of Study 2 findings is unlikely given that the learning of new words is a relatively difficult task, which according to social facilitation research should be hampered by the presence of more similar others (Geen, 1991). Still, in Study 3, I further examine whether psychological states such as positive affect, negative affect, perceived arousal, and feelings of competitiveness differ across conditions.

\section{Limitations}

In the two studies presented, it is not the mere presence of more (vs. less) similar others that was hypothesized to produce the effect but rather the fact that more (vs. less) similar others were experiencing the same stimuli available to the participant. Yet the experimental strategy utilized thus far has not distinguished between the mere presence of more (vs. less) similar others and the stimuli experiences of more (vs. less) similar others who are present. Accordingly, in Study 3, I conduct a more stringent test of the key hypothesis by including a new condition in which it is implied that more similar others are present but are experiencing a different stimulus from the one available to the participant.

\section{Study 3}

\section{Method}

Participants and general design. Participants were 44 undergraduates $(59.1 \%$ women, $40.9 \%$ men; mean age $=18.95$ years) . In addition to the two more (vs. less) similarity conditions included in previous studies, Study 3 included a new similar others condition where participants were informed that they would each see a different stimulus. The new condition was meant to make participants aware of the similar others' presence but not of the fact that the similar others were experiencing the stimulus available to the participant. As such, I expected that participants in this more similar others-different stimuli condition would exhibit less stimulus accessibility than participants in the more similar others-same 
stimuli condition and comparable stimulus accessibility to participants in the less similar others-same stimuli condition. The stimulus was a painting shown at the very beginning of the study, before participants completed a number of tasks that were extraneous to the goal of the study (i.e., filling out scales). The painting was identical to the one used in the distractor task of Study 2. It was expected that by the end of the study, participants in the more similar others-same stimulus condition would exhibit a better recognition memory for the painting than participants in the other two conditions.

Procedure and manipulations. Participants arrived at the laboratory three at a time and were placed into separate rooms, with each individual seated in front of a computer. In addition to the more similar and less similar conditions in Study 2, Study 3 included a third condition. In the third condition, although participants saw that two other participants chose the same avatar colors as they did (identical to the similar others condition), it was implied that each participant would see one of three possible paintings by clicking on a "painting" button above their own avatar, whereas in the other two conditions it was implied that all participants would see the same painting by clicking on a common "painting" button. In reality, participants across all conditions saw the same abstract painting. Participants were then asked the extent to which they liked the painting. The dependent variable of interest, however, came toward the end of the study when participants were shown a subtly different painting and were asked whether it was identical to the painting from the beginning of the study $(1=$ yes, it is identical, $2=n o$, it is different). The second painting was altered so that details that were on the left side of the first painting were now shifted to the right side and vice versa. The change was subtle and was made purposefully difficult to recognize as to prevent ceiling effects (correct recognition rate $=18 \%$ ).

Participants also filled out a Positive Affect Negative Affect Scale (Watson, Clark, \& Tellegen, 1988) and a Perceived Arousal Scale (Anderson, Deuser, \& DeNeve, 1995; positive affect $\alpha=$ .78; negative affect $\alpha=.88$; arousal scale $\alpha=.89$ ). Finally, participants were asked to what extent they felt that they were competing with the other participants $(1=$ not at all, $7=$ very much so).

\section{Results and Discussion}

Because the dependent variable was dichotomous, I used binary logistic regression to analyze the data. The effect of condition on recognition accuracy was significant, $\chi^{2}(2)=7.91, p<.05$, Cox-Snell $R^{2}=.16$. Planned contrasts indicated that participants in the more similar others-same stimulus condition $(n=14)$ had significantly different recognition accuracy rates from (a) participants in the more similar others-different stimulus condition $(n=$ 15; $\beta=2.35$, Wald $=4.06, p<.05$ ) and (b) participants in the less similar others-same stimulus condition $(n=15 ; \beta=2.35$, Wald $=4.06, p<.05$ ). Indeed, the magnitude of the differences was identical because participants in the latter two conditions had the same average accuracy rate of $6.7 \%(M=1.07, S D=0.26)$, whereas participants at the more similar others-same stimulus condition had an accuracy rate of $42.9 \%(M=1.43, S D=0.51)$.

Finally, an ANOVA indicated that participants' positive affect, $F(2,41)=0.03, p=.97$, negative affect, $F(2,41)=0.58, p=.56$, and perceived arousal, $F(2,41)=0.31, p=.74$, did not differ across the three conditions. That is, participants in the more similar others-same stimulus condition did not report greater positive affect, negative affect, or arousal (positive affect: $M=2.65, S D=$ 0.61 ; negative affect: $M=1.41, S D=0.51$; arousal: $M=3.14$, $S D=0.56$ ) than participants in the more similar others-different stimulus condition (positive affect: $M=2.72, S D=0.87$; negative affect: $M=1.57, S D=0.47$; arousal: $M=3.05, S D=0.87$ ) or participants in the less similar others-same stimulus condition (positive affect: $M=2.69, S D=0.77$; negative affect: $M=1.44$, $S D=0.34$; arousal: $M=3.25, S D=0.63$ ).

It is interesting that an ANOVA did reveal a significant difference between conditions in self-reported feelings of competitiveness, $F(2,41)=3.37, p<.05$. There were greater feelings of competitiveness in the less similar others-same stimulus condition $(M=2.47, S D=1.77)$ than (a) in the more similar others-same stimulus condition $(M=1.50, S D=0.76)$, and $(b)$ in the more similar others-different stimulus condition $(M=1.40, S D=$ 0.91). Because the variation patterns in competitiveness and painting recognition accuracy differed across conditions, the competitiveness account is not a viable alternative explanation for the key findings.

\section{Discussion}

As predicted, recognition accuracy was the highest in the condition where the original painting was assumed to be experienced by others who were more (vs. less) similar to the self. Critically, the effect did not materialize when a distinct painting was assumed to be experienced by more (vs. less) similar others. These findings provide further support for the key hypothesis: Stimuli assumed to be experienced by one's social group are rendered more cognitively accessible. Moreover, this study further limits the possibility that the observed effects can be due to the manipulation of psychological states such as positive affect, negative affect, arousal, and competitiveness. Aside from the null results on these measures, such psychological state explanations suggest that participation of more (vs. less) similar others in the same general task would have increased recognition accuracy. Study 3 results contradict this hypothesis. Indeed, the social tuning hypothesis uniquely predicts that beyond participating in the same general task, an increase in the cognitive accessibility of a stimulus results from the assumption that more (vs. less) similar others are experiencing the same exact stimulus that is available to the self.

\section{General Discussion}

Findings across three studies suggest that stimuli such as time pressure (Study 1), words (Study 2), and paintings (Study 3) are rendered more psychologically and behaviorally prominent when they are thought to be experienced by others who are more (vs. less) similar to the self. Critically, the effect is absent when more similar others are thought to be looking at a distinct stimulus (Study 3). Taken as a whole, these results are consistent with the hypothesis that stimuli that are assumed to be experienced by one's social group have greater cognitive accessibility. In what follows, I discuss the implications of this research.

\section{The Emergence of Cultural Memory}

At the outset, cultural knowledge was defined as knowledge that is shared within a social group. Further, I argued that whereas the 
psychological mechanisms behind shared attitudes have been extensively explored, the psychological mechanisms behind shared memories are still poorly understood. The findings reported here begin to outline the process by which individuals coordinate their personal memories with that of their group(s), even in the absence of intragroup communication. The results suggest that individual to group alignment is, in part, a result of what can be referred to as a fast and frugal heuristic (Gigerenzer, 2004) - if my social group experiences the stimulus, then the stimulus is mentally prioritized. Although it is possible that other psychological variables may also play an important role in the effect, at this point the above heuristic is the most parsimonious explanation that is in concert with empirical data.

It may be argued that the studies presented do not show the creation of actual shared memories. This is true, because the studies' participants tuned into the stimuli experiences of nonexistent groups and as such, actual social coordination did not occur (with the exception of decision speed in Study 1). However, it is important to remember that the creation of nonexistent groups in participants' minds served as an experimental technique that lent greater internal validity to the test of the proposed psychological mechanism. That is, the presence of actual group members would have allowed other interpretations of our findings (e.g., verbal communication, gaze following, behavioral mimicry). Notably, the fact that participants tuned into the stimuli experiences of nonexistent social groups that they thought existed allows for the conclusion that the effect would be the same for existent social groups (as long as they were thought to exist).

\section{Of Colors and Avatars}

The findings presented are also noteworthy due to the minimal nature of the manipulations utilized. Beyond the methodological benefits of using minimal manipulations (i.e., avoiding alternative interpretations), there are also significant theoretical benefits to using minimal manipulations. First, across all participant debriefings, there was not one instance when a participant expressed the suspicion that they were influenced by the avatar choices of others. Upon hearing the hypothesis being tested, the vast majority of participants expressed wonder at the idea that such a minimal manipulation would influence their thoughts and actions. As such, the findings show that people's cognitions and behaviors are more affected by their social groups than they imagine. Given that everyday experiences are infused with far stronger social group contexts than utilized in these experiments, it is conceivable that large portions of what people think of as their individual memories are a product of social tuning.

\section{Social Coordination in Silence}

The findings shed some light on the dynamics of social coordination. First and foremost, social groups' capacity to maintain social coordination is not entirely dependent on the use of language. Rather, as the findings show, group members' assumptions about one another's stimuli experiences can lead to greater social tuning. In other words, the proposed psychological mechanism allows for the emergence of culture under seemingly asocial circumstances, in the absence of actual social interaction. It is thus possible that social attunement within large, abstract groups (e.g., nations, organizations, schools) as well as small, face-to-face groups (e.g., families, friends, teams) is maintained by way of vigilant monitoring for stimuli that are experienced by the group. Indeed, I speculate that the ease of actual intragroup communication may be largely a product of the silent social tuning processes that precede it.

\section{Unanswered Questions}

The social tuning hypothesis allows for many avenues of future research. First, it is important to examine the cognitive processes that underpin the effects found. For instance, it is possible that stimuli that are thought to be experienced by one's social group receive greater attentional focus, which increases their cognitive accessibility. It is also conceivable that stimuli that are thought to be experienced by one's social group receive more elaborated encoding, which increases their cognitive accessibility. Alternatively, it is possible that such social stimuli are stored in memory centers where retrieval is more likely.

Second, it is important to examine the motivational processes underpinning social tuning. At this point, it is unclear whether individuals have an explicit or an implicit goal to attend to and remember stimuli that are thought to be experienced by their social group. Given that participants doubted the influence of others on their cognition and behavior, I believe it is somewhat unlikely that they had an explicit goal. Rather, it is more probable that social tuning is a highly implicit process. Relatedly, more research is needed to ascertain whether participants in the reported studies experienced a shared inner state (Hardin \& Higgins, 1996), as well as whether an experience of such a state is instrumental to the formation of shared memories.

Third, it is important to examine how group size influences the cognitive accessibility of a stimulus. That is, does the cognitive accessibility of a stimulus depend on whether it is thought to be experienced by a larger (vs. smaller) social group? The answer to this question may have particularly interesting implications in today's world of social media, where individuals are routinely aware that what they see, read, and hear is being experienced by millions of others, who may or may not be their group members.

\section{Conclusion}

The social tuning hypothesis offers a novel approach to understanding the emergence of culture. Although no theory can be proved certain, the three studies presented constitute initial support. The results of these studies as well as future explorations of the topic are likely to have important implications for social learning, social coordination, and social behavior across schools and living rooms - or any social context where stimuli are thought to be experienced by others.

\section{References}

Anderson, C. A., Deuser, W. E., \& DeNeve, K. M. (1995). Hot temperatures, hostile affect, hostile cognition, and arousal: Tests of a general model of affective aggression. Personality and Social Psychology Bulletin, 21, 434-448.

Asch, S. E. (1952). Social psychology. New York, NY: Prentice-Hall.

Banks, W. P. (1970). Signal detection theory and human memory. Psychological Bulletin, 74, 81-99. 
Baron-Cohen, S. (1995). Mindblindness: An essay on autism and theory of mind. Cambridge, MA: MIT Press.

Broadbent, D. (1958). Perception and communication. London, England: Pergamon Press.

Bruner, J. S. (1957). On perceptual readiness. Psychological Review, 64, 123-152.

Carpenter, M., Nagell, K., \& Tomasello, M. (1998). Social cognition, joint attention, and communicative competence from 9 to 15 months of age. Monographs of the Society for Research in Child Development, 4, $1-143$.

Clark, H. H. (1996). Using language. Cambridge, MA: Cambridge University Press.

Clark, H. H. (2003). Pointing and placing. In S. Kita (Ed.), Pointing: Where language, culture, and cognition meet (pp. 243-268). Hillsdale NJ: Erlbaum.

Cosmides, L., Tooby, J., \& Barkow, J. H. (1992). Introduction: Evolutionary psychology and conceptual integration. In J. H. Barkow, L. Cosmides, \& J. Tooby (Eds.), The adapted mind: Evolutionary psychology and the generation of culture (pp. 3-15). New York, NY: Oxford University Press.

Durkheim, E. (1984). The division of labor in society. New York, NY: Free Press. (Original work published 1893)

Echterhoff, G., Higgins, E. T., \& Levine, J. M. (2009). Shared reality experiencing commonality with others' inner states about the world. Perspectives on Psychological Science, 4, 496-521.

Fazio, R. H. (1989). On the power and functionality of attitudes: The role of attitude accessibility. In A. R. Pratkanis, S. J. Breckler, \& A. G. Greenwald (Eds.), Attitude structure and function (pp. 153-179). Hillsdale, NJ: Erlbaum.

Festinger, L. (1950). Informal social communication. Psychological Review, 57, 271-282.

Festinger, L. (1957). A theory of cognitive dissonance. Evanston, IL: Row, Peterson.

Geen, R. G. (1991). Social motivation. Annual Review of Psychology, 42, 377-399.

Gigerenzer, G. (2004). Fast and frugal heuristics: The tools of bounded rationality. In D. D. Koehler \& N. Harvey (Eds.), Blackwell handbook of judgment and decision making (pp. 62-88). Oxford, England: Blackwell.

Hardin, C. D., \& Higgins, E. T. (1996). Shared reality: How social verification makes the subjective objective. In E. T. Higgins \& R. M. Sorrentino (Eds.), Handbook of motivation and cognition: The interpersonal context (Vol. 3, pp. 28-84). New York, NY: Guilford Press.

Higgins, E. T., \& King, G. A. (1981). Accessibility of social constructs: Information-processing consequences of individual and contextual variability. In N. Cantor \& J. F. Kihlstrom (Eds.), Personality, cognition, and social interaction (pp. 69-121). Hillsdale, NJ: Erlbaum.
Hirst, W., \& Echterhoff, G. (2008). Creating shared memories in conversation: Towards a psychology of collective memory. Social Research, $75,78-91$.

James, W. (1907). Pragmatism's conception of truth. The Journal of Philosophy Psychology and Scientific Method, 4, 144-155.

Kroeber, A., \& Kluckhohn, C. (1952). Culture. New York, NY: Meridian Books.

Lewin, K. (1943). Defining the 'field at a given time.' Psychological Review, 50, 292-310.

Mead, G. H. (1934). Mind, self and society. Chicago, IL: University of Chicago Press.

Miller, A. G. (1956). The magical number seven, plus or minus two. Psychological Review, 2, 81-97.

Morales, M., Mundy, P., Delgado, C. E. F., Yale, M., Messinger, D., Neal, R., \& Schwartz, H. K. (2000). Responding to joint attention across the 6- through 24-month age period and early language acquisition. Journal of Applied Developmental Psychology, 21, 283-298.

Sechrist, G. B., \& Stangor, C. (2001). Perceived consensus influences intergroup behavior and stereotype accessibility. Journal of Personality and Social Psychology, 80, 645-654.

Sober, E., \& Wilson, D. S. (1998). Unto others: The evolution and psychology of unselfish behavior. Boston, MA: Harvard University Press.

Tomasello, M., Carpenter, M., Call, J., Behne, T., \& Moll, H. (2005). Understanding and sharing intentions: The origins of cultural cognition. Behavioral and Brain Sciences, 28, 675-735.

Turner, J. C. (1991). Social influence. Milton-Keynes, England: Open University Press.

Turner, J. C. (1999). Some current issues in research on social identity and self-categorization theories. In N. Ellemers, R. Spears, \& B. Doosje (Eds.), Social identity: Context, commitment, content (pp. 6-34). Oxford, England: Blackwell.

Turner, J. C., Hogg, M. A., Oakes, P. J., Reicher, S. D., \& Wetherell, M. S. (1987). Rediscovering the social group: A self-categorization theory. New York, NY: Blackwell.

Vygotsky, L. S. (1978). Mind in society: The development of higher psychological processes. Cambridge, MA: Harvard University Press.

Watson, D., Clark, L. A., \& Tellegen, A. (1988). Development and validation of brief measures of positive and negative affect: The PANAS scales. Journal of Personality and Social Psychology, 54, 1063-1070.

Wilson, D. S., \& Wilson, E. O. (2007). Rethinking the theoretical foundation of sociobiology. Quarterly Review of Biology, 82, 327-348.

Received November 30, 2009

Revision received February 12, 2010

Accepted February 16, 2010 